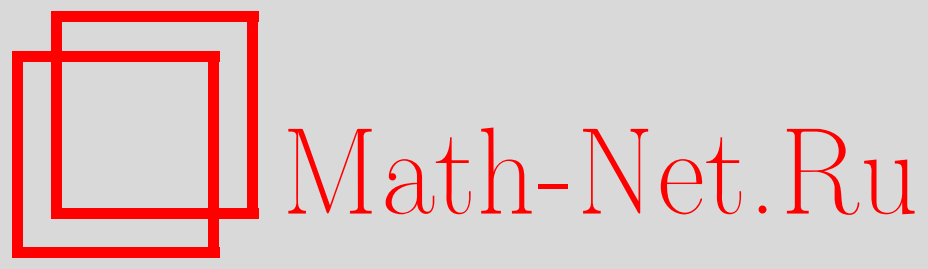

Г. С. Мустафаев, Слабо компактные гомоморфизмы регулярных банаховых алгебр, Функи. анализ и его прил., 2008, том 42, выпуск 2, 85-89

DOI: https://doi.org/10.4213/faa2907

Использование Общероссийского математического портала MathNet.Ru подразумевает, что вы прочитали и согласны с пользовательским соглашением

http://www.mathnet.ru/rus/agreement

Параметры загрузки:

IP : 3.89 .185 .249

26 апреля 2023 г., 16:06:31

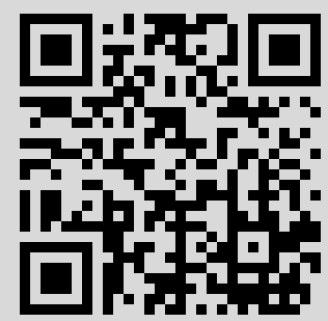


УДК 517.98

\title{
Слабо компактные гомоморфизмы регулярных банаховых алгебр
}

\author{
(c) 2008. Г. С. МустаФАев
}

Посвящается 70-летию профессора А. Д. Гаджиева

В этой заметке изучается структура слабо компактных гомоморфизмов банаховых алгебр.

Пусть $A$ - комплексная коммутативная банахова алгебра, а $M_{A}$ - пространство ее максимальных идеалов. Через $\hat{a}$ будем обозначать преобразование Гельфанда элемента $a \in A$. Если алгебра $A$ не имеет единичного элемента, то алгебру, полученную из нее присоединением единицы, будем обозначать через $A_{1}$. Хорошо известно, что $M_{A_{1}}=M_{A} \cup\left\{\phi_{\infty}\right\}$ является одноточечной компактификацией пространства $M_{A}$. Алгебра $A$ называется регулярной (по Шилову) ([1, гл. 6], [2]), если для каждого $\phi \in M_{A}$ и замкнутого подмножества $S \subset M_{A}$, такого, что $\phi \notin S$, существует элемент $a \in A$, такой, что $\hat{a}(\phi)=1$ и $\hat{a}(S)=\{0\}$. Хорошо известно, что регулярные банаховы алгебры обладают свойством нормальности: для каждого компакта $K \subset M_{A}$ и замкнутого подмножества $S \subset M_{A}$, такого, что $K \cap S=\varnothing$, существует элемент $a \in A$, удовлетворяющий условиям $\hat{a}(K)=\{1\}$ и $\hat{a}(S)=\{0\}$. Однако «управлять» нормой элемента $a$, вообще говоря, невозможно.

Регулярную банахову алгебру $A$ будем называть равномерно регулярной, если существует такая константа $C>0$, что для каждого компакта $K \subset M_{A}$ и $\phi \in M_{A} \backslash K$ найдется элемент $a \in A$, для которого $\hat{a}(\phi)=0, \hat{a}(K)=\{1\}$ и $\|a\| \leqslant C$.

Пусть $X$ - локально компактное хаусдорфово пространство и $C_{0}(X)$ - банахова алгебра всех непрерывных комплексных функций на $X$, стремящихся к нулю на бесконечности. В силу леммы Урысона $C_{0}(X)$ равномерно регулярна.

Коммутативная банахова алгебра $A$ называется ограниченно регулярной ([3, c. 418], [4]), если она удовлетворяет следующим условиям: существует такая константа $C>0$, что для каждой точки $\phi \in M_{A}$ и каждой окрестности $U$ этой точки найдется элемент $a \in A$, для которого выполняются условия $\hat{a}(\phi)=1$, supp $\hat{a} \subset U$ и $\|a\| \leqslant C$.

Пусть $A$ - регулярная полупростая банахова алгебра. Положим

$$
A_{00}=\{a \in A: \operatorname{supp} \hat{a} \text { компактен }\} .
$$

Kак известно [1, гл. 6], для произвольного $\phi \in M_{A}$ множество $I_{\phi}=\{a \in A$ : $\hat{a}(\phi)=0\}$ образует наибольший замкнутый идеал в $A$ с оболочкой $\{\phi\}$, а $J_{\phi}=$ $\overline{J_{\phi}^{0}}$ - наименьший замкнутый идеал в $A$ с оболочкой $\{\phi\}$, где $J_{\phi}^{0}=\left\{a \in A_{00}: \phi \notin\right.$ $\operatorname{supp} \hat{a}\}$. Заметим, что $I_{\phi_{\infty}}=A$ и $J_{\phi_{\infty}}^{0}=A_{00}$. Алгебра $A$ называется алгеброй Диткина, если каждая точка из $M_{A}$ (а в случае, когда $A$ не имеет единичного элемента, и $\left.\phi_{\infty}\right)$ является множеством синтеза для $A$ (для $A_{1}$ ). Таким образом, $A$ является алгеброй Диткина тогда и только тогда, когда $I_{\phi}=J_{\phi}$ для всех $\phi \in M_{A}$ и $\overline{A_{00}}=A$. 
Пример 1. Если $G$ - компактная абелева группа, то $A=L^{2}(G)$ со сверткой в качестве умножения и обычной нормой является коммутативной банаховой алгеброй. Отметим, что $M_{A}=\widehat{G}$, где $\widehat{G}-$ группа характеров группы $G$. Преобразование Гельфанда элемента $f \in L^{2}(G)$ совпадает с его преобразованием Фурье $\hat{f}$. Так как $M_{A}$ дискретно, то алгебра $A$ регулярна. Нетрудно заметить, что $A$ - ограниченно регулярная алгебра Диткина. Однако равенство Парсеваля

$$
\|f\|_{2}^{2}=\sum_{\chi \in \widehat{G}}|\hat{f}(\chi)|^{2}, \quad f \in L^{2}(G),
$$

показывает, что $A$ не является равномерно регулярной. Заметим также, что она не имеет ограниченной аппроксимативной единицы (о.а.е).

Предложение 2. Пусть $A$ - регулярнал банахова алгебра с о.а.е. Если $A$ ограниченно регулярна, то она равномерно регулярна.

Доказательство. Предположим, что алгебра $A$ имеет аппроксимативную единицу, ограниченную некоторой константой $C>0$. Пусть $K-$ компактное подмножество в $M_{A}$ и $\phi \in M_{A} \backslash K$. Так как алгебра $A$ регулярна, то существует такой элемент $a \in A$, что $\hat{a}(K)=\{1\}$. В силу теоремы факторизации Коэна-Хьюитта $[5,32.22]$ существуют элементы $a_{1}, a_{2} \in A$, такие, что $a=a_{1} a_{2}$, $\left\|a_{1}-a\right\| \leqslant 1$ и $\left\|a_{2}\right\| \leqslant C$. Пусть $b=a+a_{2}-a a_{2}=a_{2}+\left(a_{1}-a\right) a_{2}$. Тогда $\hat{b}(K)=\{1\}$ и $\|b\| \leqslant 2 C$. С другой стороны, поскольку $\phi \notin K$, имеется такая окрестность $U$ точки $\phi$, что $U \cap K=\varnothing$. Так как алгебра $A$ ограниченно регулярна, то существует константа $L>0$ и элемент $d \in A$, для которых выполняются условия $\hat{d}(\phi)=1, \operatorname{supp} \hat{d} \subset U$ и $\|d\| \leqslant L$. Положим $h=b-d b$. Тогда $\hat{h}(\phi)=\hat{b}(\phi)-\hat{d}(\phi) \hat{b}(\phi)=0, \hat{h}(K)=\hat{b}(K)-\hat{d}(K) \hat{b}(K)=\hat{b}(K)=\{1\}$ и $\|h\| \leqslant(1+\|d\|)\|b\| \leqslant 2 C(1+L)$.

Пусть $G$ - локально компактная группа, и пусть $A_{p}(G)(1<p<\infty)-$ пространство функций $f$ на $G$, представимых в виде

$$
f=\sum_{n=1}^{\infty} u_{n} * v_{n}^{\vee},
$$

где $\left\{u_{n}\right\}_{n \in \mathbb{N}} \subset L^{p}(G),\left\{v_{n}\right\}_{n \in \mathbb{N}} \subset L^{q}(G)(1 / p+1 / q=1), v_{n}^{\vee}(g)=v_{n}(-g)$ и

$$
\|f\|=\inf \sum_{n=1}^{\infty}\left\|u_{n}\right\|_{p}\left\|v_{n}\right\|_{q}<\infty ;
$$

здесь инфимум берется по всевозможным представлениям функции $f$. Тогда $A_{p}(G)$ есть коммутативная регулярная банахова алгебра относительно обычного умножения, часто называемая алгеброй Герца [6]. Более того, $A_{p}(G)$ является алгеброй Диткина [6]. Как замечено в [4], она ограниченно регулярна. С другой стороны, в случае когда группа $G$ аменабельна, $A_{p}(G)$ имеет о.а.е. [6]. Теперь, применяя предложение 2 , получаем такое

Предложение 3. Если $G$ - аменабельная группа, то алгебра $A_{p}(G)$ равномерно регулярна.

Заметим, что если $G$ - абелева группа, то $L^{1}(G) \cong A_{2}(\widehat{G})$ и потому $L^{1}(G)-$ равномерно регулярная алгебра Диткина. 
Следующий пример показывает, что без условия аменабельности предложение 3 перестает быть верным.

Пример 4. Пусть $G$ - дискретная группа, содержащая свободную группу $F_{2}$ с двумя образующими. Хорошо известно, что $G$ неаменабельна. Кроме того, она содержит такое бесконечное множество $S$ (множество Лейнерта), что пространство $M=\left\{\varphi \in A_{2}(G)^{*}: \operatorname{supp} \varphi \subseteq S\right\}$ изоморфно (по норме) пространству $l^{2}(S)$ [7]. Отсюда следует, что пространство $M$ является сопряженным пространством пространства $A_{2}(G) / J_{S}$, где $J_{S}$ - наименьший замкнутый идеал алгебры $A_{2}(G)$ с оболочкой $S$. Таким образом, $A_{2}(G) / J_{S}$ изоморфно пространству $l^{2}(S)$. Следовательно, существует такая константа $C>0$, что при Bcex $f \in A_{2}(G)$

$$
\|f\|^{2} \geqslant\|f+J\|^{2} \geqslant C \sum_{g \in S}|f(g)|^{2} .
$$

Это показывает, что алгебра $A_{2}(G)$ не является равномерно регулярной.

Пусть $B V(\mathbb{R})$ - пространство всех комплексных функций на $\mathbb{R}$ с ограниченным изменением. Положим $B V C_{0}(\mathbb{R})=C_{0}(\mathbb{R}) \cap B V(\mathbb{R})$. Тогда относительно обычного умножения и нормы

$$
\|f\|=\|f\|_{\infty}+\operatorname{Var}_{\mathbb{R}}(f)
$$

$B V C_{0}(\mathbb{R})$ становится регулярной банаховой алгеброй. Пространство максимальных идеалов этой алгебры отождествляется с $\mathbb{R}$ [8, следствие 3.2.8]. Нетрудно заметить, что алгебра $B V C_{0} \mathbb{R}$ ) ограниченно регулярна и имеет о.а.е. Более того, $B V C_{0}(\mathbb{R})$ - алгебра Диткина (доказательство этого факта подобно доказательству из [8, с. 302, А.2.5]). Теперь, применяя предложение 2, получаем такое

Предложение 5. $B V C_{0}(\mathbb{R})$ является равномерно регулярной банаховой алгеброй.

В работах [9] и [10, следствие 3.7] доказано, что если $G$ - локально компактная аменабельная группа, то любой слабо компактный гомоморфизм из $A_{p}(G)$ в произвольную банахову алгебру является конечномерным.

Основным результатом этой заметки является следующая теорема.

Теорема 6. Пусть $A$ - равномерно регулярная алгебра Диткина, а $B$ произвольная банахова алгебра (не обязательно коммутативная). Тогда любой слабо компактный гомоморфизм из $A$ в $B$ является конечномерным.

Для доказательства нам понадобятся следующие леммы.

Лемма 7. Пусть - равномерно регулярная алгебра Диткина. Тогда для произвольного $\phi \in M_{A} \cup\left\{\phi_{\infty}\right\}$ идеал $I_{\phi}(u$, в частности, алгебра $A)$ имеет o.a.e.

Доказательство. Пусть $\phi \in M_{A} \cup\left\{\phi_{\infty}\right\}$ и $K_{\phi}-$ направленное (по включению) множество компактных подмножеств пространства $M_{A}$, не содержащих точку $\phi$. Так как алгебра $A$ равномерно регулярна, то существует такая константа $C>0$, что для каждого $K \in K_{\phi}$ найдется элемент $a_{K} \in A$, такой, что $\hat{a}_{K}(\phi)=0, \hat{a}(K)=\{1\}$ и $\left\|a_{K}\right\| \leqslant C$. Возьмем произвольный элемент $b \in J_{\phi}^{0}$. Так как $\phi \notin \operatorname{supp} \hat{b}$, то $\operatorname{supp} \hat{b} \subset K$ для некоторого $K \in K_{\phi}$ и поэтому $a_{K} b=b$. Поскольку $J_{\phi}^{0}$ плотно в $I_{\phi}$, направленность $\left\{a_{K}\right\}_{K \in K_{\phi}}$ служит о.а.е. для $I_{\phi}$. 
Лемма 8. Пусть $A$ - равномерно регулярная алгебра Диткина. Если $S$ является относительно слабо компактным подмножеством в $M_{A}$, то оно конечно.

Доказательство. Предположим, что $S$ - бесконечное множество. Тогда существует такая последовательность $\left\{\phi_{n}\right\}_{n \in \mathbb{N}}$ попарно различных элементов множества $S$, что $\left\{\phi_{n}\right\}$ слабо сходится к $\phi$. Нетрудно видеть, что $\phi \in M_{A} \cup$ $\left\{\phi_{\infty}\right\}$. В силу леммы 7 алгебра $A$ имеет о.а.е. Отсюда и из $[10$, предложение 2.8] следует, что $M_{A}$ слабо замкнуто и поэтому $\phi \in M_{A}$. Тогда для любого $\varepsilon>0$ существует такая выпуклая комбинация элементов $\phi_{n_{1}}, \ldots, \phi_{n_{k}} \in M_{A}$, что $\left\|\phi-\lambda_{1} \phi_{n_{1}}-\cdots-\lambda_{k} \phi_{n_{k}}\right\|<\varepsilon$, где $\lambda_{i}>0(i=1, \ldots, k)$ и $\lambda_{1}+\cdots+\lambda_{k}=1$. Так как алгебра $A$ равномерно регулярна, то существует такая константа $C>0$ и такой элемент $a \in A$, что $\hat{a}(\phi)=0, \hat{a}\left(\phi_{n_{1}}\right)=\cdots=\hat{a}\left(\phi_{n_{k}}\right)=1$ и $\|a\| \leqslant C$. Следовательно,

$$
\left|\hat{a}(\phi)-\lambda_{1} \hat{a}\left(\phi_{n_{1}}\right)-\cdots-\lambda_{k} \hat{a}\left(\phi_{n_{k}}\right)\right|<\varepsilon\|a\| \leqslant \varepsilon C,
$$

так что $1<\varepsilon C$. То есть мы пришли к противоречию.

Доказательство теоремы 6. Пусть $h: A \rightarrow B$ - слабо компактный гомоморфизм. Без ограничения общности можно считать, что $B=\overline{h(A)}$ и алгебра $B$ коммутативна. Пусть $\psi \in M_{B} \cup\left\{\psi_{\infty}\right\}$ и $h^{*} \psi=\phi$ (очевидно, что $\left.h^{*} \psi_{\infty}=\phi_{\infty}\right)$. Положим $I_{\psi}=\{b \in B: \hat{b}(\psi)=0\}$ и покажем, что $I_{\psi}=\overline{h\left(I_{\phi}\right)}$. Так как $\overline{h\left(I_{\phi}\right)} \subset I_{\psi}$, то достаточно доказать обратное включение. С этой целью возьмем произвольное $\varepsilon>0$ и $b \in I_{\psi}$. Тогда $\|b-h(a)\| \leqslant \varepsilon$ для некоторого $a \in A$, откуда следует, что $|\hat{a}(\phi)| \leqslant \varepsilon$. Далее, существует такой элемент $a_{\phi} \in A$, что $\hat{a}_{\phi}(\phi)=1$ и $\left\|a_{\phi}\right\| \leqslant 2$. Если $d=a-\hat{a}(\phi) a_{\phi}$, то $d \in I_{\phi}$ и $\|b-h(d)\|=\left\|b-h(a)+\hat{a}(\phi) h\left(a_{\phi}\right)\right\| \leqslant \varepsilon+2 \varepsilon\|h\|$.

В силу леммы 7 идеал $I_{\phi}$ имеет о.а.е. $\left(e_{\lambda}\right)_{\lambda \in \Lambda}$. Так как отображение $h$ слабо компактно, без ограничения общности можно считать, что направленность $\left\{h\left(e_{\lambda}\right)\right\}$ слабо сходится к $e$ для некоторого $e \in B$. Легко заметить, что $e \in I_{\psi}$. Мы видим, что, с одной стороны, направленность $\left\{h(a) h\left(e_{\lambda}\right)\right\}$ слабо сходится к $h(a) e$ при всех $a \in A$, а с другой стороны, $\left\{h(a) h\left(e_{\lambda}\right)\right\}$ сходится к $h(a)$ по норме при всех $a \in I_{\phi}$. Следовательно, $h(a) e=h(a)$ при всех $a \in I_{\phi}$. В силу плотности $h\left(I_{\phi}\right)$ в $I_{\psi}$ получаем, что $b e=b$ при всех $b \in I_{\psi}$. Таким образом, е является идемпотентом в $I_{\psi}$ и потому $I_{\psi}=e B$.

Нетрудно видеть, что $h^{*} M_{B} \subset M_{A}$. Поэтому $h^{*} M_{B}$ является относительно слабо компактным подмножеством в $M_{A}$. В силу леммы 8 это конечное множество, $h^{*} M_{B}=\left\{\phi_{1}, \ldots, \phi_{n}\right\}$. Так как $h^{*}$ инъективно, $M_{B}=\left\{\psi_{1}, \ldots, \psi_{n}\right\}$. Как мы уже заметили выше, $I_{\psi_{i}}=e_{i} B(i=1, \ldots, n)$, где $e_{i}$ - некоторый идемпотент в $I_{\psi_{i}}$. Таким образом, $\operatorname{Rad}(B)=\bigcap I_{\psi_{i}}=e B$, где $e=e_{1} \cdots e_{n}$. Так как $e^{2}=e$ и $e \in \operatorname{Rad}(B)$, то $e=0$. Таким образом, $B-$ полупростая банахова алгебра, пространство максимальных идеалов которой конечно. Следовательно, алгебра $B$ конечномерна.

Следующий пример показывает, что без условия равномерной регулярности теорема 6 перестает быть верной.

Пример 9. Пусть $G$ - бесконечная компактная абелева группа. Как мы уже заметили выше, $L^{2}(G)$ - алгебра Диткина, но она не является равномерно регулярной. Однако id: $L^{2}(G) \rightarrow L^{2}(G)$ есть бесконечномерный слабо компактный гомоморфизм. 
Следствие 10. Пусть $A-$ равномерно регулярная алгебра Диткина, а $X-$ замкнутый по норме $A$-подмодуль пространства $A^{*}$. Если $X$-рефлексивное пространство, то оно конечномерно.

\title{
ЛитерАТУРА
}

[1] И. М. Гельфанд, Д. А. Райков, Е. Г. Шилов, Коммутативные нормированные кольца, Физматлит, М., 1960. [2] Е. А. Горин, УМН, 33:4 (1978), 169-188. [3] K. B. Laursen, M. Neumann, An Introduction to Local Spectral Theory, The Clarendon Press, Oxford Univ. Press, 2000. [4] A. Ülger, Studia Math., 153:1 (2002), 59-80. [5] Э. Хьюитт, К. Росс, Абстрактный гармонический анализ, т. 2, Наука, М., 1975. [6] C. Herz, Ann. Inst. Fourier, 23 (1973), 91-123. [7] M. Leinert, Studia Math., 52 (1974), 149-158. [8] C. Rickart, General Theory of Banach Algebras, Van Nostrand, Princeton, NJ, 1960. [9] E. E. Granirer, Math. Japonica, 46:1 (1997), 69-72. [10] A. Ülger, Monatsh. Math., 121:4 (1996), 353-379. [11] J. E. Gale, T. J. Ransford, M. C. White, Trans. Amer. Math. Soc., 331:2 (1992), 815-824. [12] E. E. Granirer, Colloq. Math., 51 (1987), 155-163.

Университет Йузунджу Йыл, Турция e-mail: hsmustafayev@yahoo.com

Поступило в редакцию 7 ноября 2006 г.

УДК $515.12+517.982 .252$

\section{Непрерывные селекции как параметрически заданные интегралы*}

\author{
(C) 2008. П. В. Семенов
}

Локальная выпуклость топологического векторного пространства (ТВП), содержащего значения отображения, является одним из решающих условий для получения положительных результатов как в теории продолжений (теорема Дугунджи), так и в теории селекций (теоремы Майкла) и в теории неподвижных точек (теоремы Тихонова, Гликсберга) отображений бесконечномерных пространств. Для теории неподвижных точек и для продолжения отображений получено значительное число результатов и при отсутствии локальной выпуклости ([5], [7], [8], [11]). В отличие от этого в теории селекций такие продвижения практически отсутствуют. Исключение составляет, пожалуй, недавняя работа Добровольского и ван Милла [6]. В настоящей заметке доказана теорема о наличии однозначных селекций для выпуклозначных отображений в ТВП, не являющиеся локально выпуклыми.

Определение 1. Семейство $\left\{C_{\alpha}\right\}_{\alpha \in A}$ выпуклых подмножеств ТВП $Y$ называется равномерно локально выпуклым, если для любой окрестности $U=U(0)$ нуля в $Y$ найдется такая окрестность $V=V(0)$ в $Y$, что для всякого индекса $\alpha \in A$ и всякого предкомпакта $B_{\alpha} \subset C_{\alpha}$ выпуклая оболочка множества $\left(B_{\alpha}-B_{\alpha}\right) \cap V$ содержится в $U$.

Грубо говоря, $V$-близость соответствующих вершин симплексов, принадлежащих $B_{\alpha}$, гарантирует $U$-близость точек этих симплексов с одинаковыми барицентрическими координатами. Для одноточечного множества индексов $A$,

*Работа поддержана грантом РФФИ 08-01-00663. 